\title{
Water-Triggered Dimensional Swelling of Cellulose Nanofibril Films: Instant Observation Using Optical Microscope
}

\author{
Yan Qing, ${ }^{1,2}$ Yiqiang Wu, ${ }^{1}$ Zhiyong Cai, ${ }^{2}$ and Xianjun $\mathrm{Li}^{1}$ \\ ${ }^{1}$ School of Materials Science and Engineering, Central South University of Forestry and Technology, Changsha, Hunan 410004, China \\ ${ }^{2}$ Forest Products Laboratory, USDA Forest Service, Madison, WI 53726-2398, USA \\ Correspondence should be addressed to Yiqiang Wu; wuyiqiang@csuft.edu.cn and Zhiyong Cai; zcai@fs.fed.us
}

Received 2 January 2013; Accepted 30 January 2013

Academic Editor: Yongfeng Luo

Copyright (c) 2013 Yan Qing et al. This is an open access article distributed under the Creative Commons Attribution License, which permits unrestricted use, distribution, and reproduction in any medium, provided the original work is properly cited.

\begin{abstract}
To understand the swelling behavior of cellulose nanofibril (CNF) films, the dimensional variation of untreated and phenol formaldehyde modified CNF (CNF/PF) films soaked in distilled water was examined in situ with microscopic image recording combined with pixel calculation. Results showed that a dramatic thickness increase exhibited in both CNF and CNF/PF films, despite being at different swelling levels. Compared to thickness swelling, however, the width expansion for these films is negligible. Such significant difference in dimensional swelling for CNF and PF modified films is mainly caused by nanofibril deposition and their mesostructure. However, addition of PF modifier has a positive effect on the constraint of water absorption and thickness swelling, which is strongly dependent on PF loadings.
\end{abstract}

\section{Introduction}

Due to global concerns of environmental protection, interest in bio-based materials is growing. As one of these subjects, cellulose nanofibril (CNF), which was created by subjecting bleached plant pulp fibers to mechanical homogenization or chemical oxidization, has attracted extensive investigation $[1,2]$. The nanofibers generated are composed primarily of microfibrils and microfibril aggregations and maintain a gel-like state even with low solid concentration in suspension. When removing excess water from CNF solution, the entangled individual nanofibrils are initially attracted by capillary force and then formed into stiff and transparent films connected via hydrogen bonds between adjacent nanofibrils [3]. The resultant films exhibit a broad range of advantages, including excellent mechanical strength, low thermal expansion $[4,5]$, high visible light transmittance, and desirable $\mathrm{O}_{2}$ and $\mathrm{CO}_{2}$ barrier capabilities [6]. These films are considered as potential candidates in various applications of packaging, medical carriers, flexible display substrates, and battery membranes [7]. However, the natural hydrophilicity and thickness swelling due to moisture and humidity have restricted CNF films from wide commercialization. For tailoring and functionally modifying CNF films aimed at a widespread utilization, the understanding of their swelling behavior and interaction with solvents and modified agents is of great importance.

However, measurements using conventional devices to directly estimate the dimensional change caused by solvent swelling are recently difficult. This is largely because of the soft characteristics of wet/swelled CNF film. Until now, quartz crystal microbalance with dissipation (QCMD) or ellipsometry (QCM-E), usually in combination with atomic force microscopy, is the most used instrument [810]. Frequently, sample films with cellulose nanofibrils are deposited or spin-coated on silica substrates prior to testing $[9,10]$. The QCM-D (E) measurement provides information such as quantity of solvent absorbed, thickness swelling, and dynamic modulus dissipation [11]. However, this technique requires that the starting materials are rigidly attached on the silicon substrate to undergo crystal oscillation in various frequencies. In order to ensure that the model films are tightly attached to their substrates, it is necessary to introduce anchoring substances to improve the adhesion between dissimilar materials [9-11]. Though highly accurate and precise, preparation of model samples is time consuming and complicated. The anchoring substance and excessive preparation time have serious negative influences on evaluating the nature 


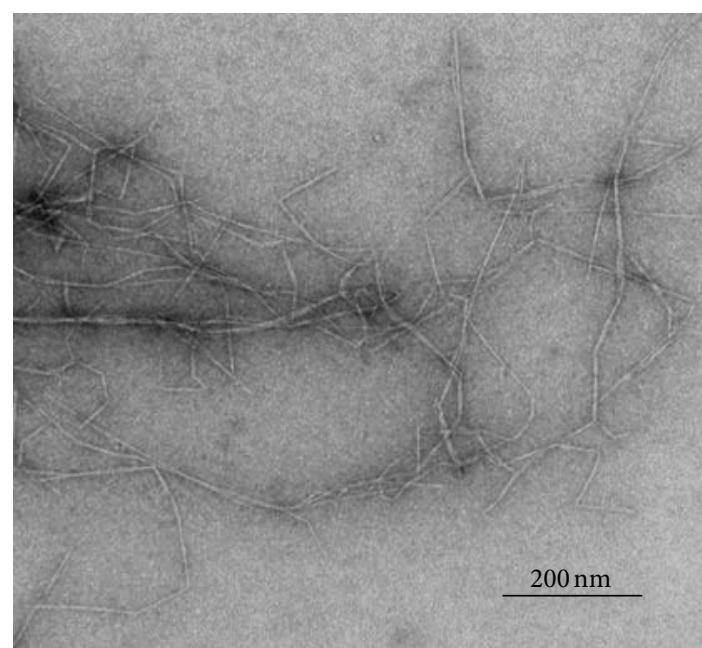

Figure 1: Typical TEM image for the TEMPO-oxidized cellulose nanofibrils.

of instantaneous swelling. Except that, QCM-D measurement hardly provides any swelling characteristic of model films in the plane orientation. However, dimensional swellings in different orientations for nanofibril films are observed significantly different in the present study. To the best of our knowledge, there are scant literature reports using other methods to monitor the instantaneous dimensional changes of CNF materials.

As a highly popular apparatus, optical microscope is efficient in measuring the dimensional change of lignincellulosic materials at high humidity or is directly impregnated into water. For instance, Perré and Huber [12] successfully measured the free shrinkage of Douglas fir and spruce wood soaked in water using an optical microscope. Taguchi et al. [13] used a similar method to investigate deformation of wood tracheid cells subjected to various moisture contents. Our cooperators have recently proposed a feasible method using the metallographic microscope at a magnification of $100 \mathrm{x}$ to measure the swelling rate of CNF films soaked in bio-based epoxy [14]. Based on this approach, a fast and easy method to instantly monitor the swelling behavior of CNF films in water was developed by using optical microscope. Dimensional changes including instantaneous thickness swelling and width expansion are recorded and calculated. Additionally, the swelling capacity of CNF composite films modified with phenol formaldehyde (PF) was estimated. It was confirmed that such information collected by microscopic images is effective and valuable for in situ measurement of swelling characteristics of CNF film in different liquids.

\section{Materials and Methods}

2.1. Preparation of TEMPO-Oxidized Cellulose Nanofibril. Cellulose nanofibrils used in this work were tetramethylpiperidine-1-oxyl (TEMPO) oxidized wood pulp fibers, which were prepared according to the method reported by Saito et al. [15] and described as follows. Commercially supplied bleached kraft eucalyptus pulps were oxidized in

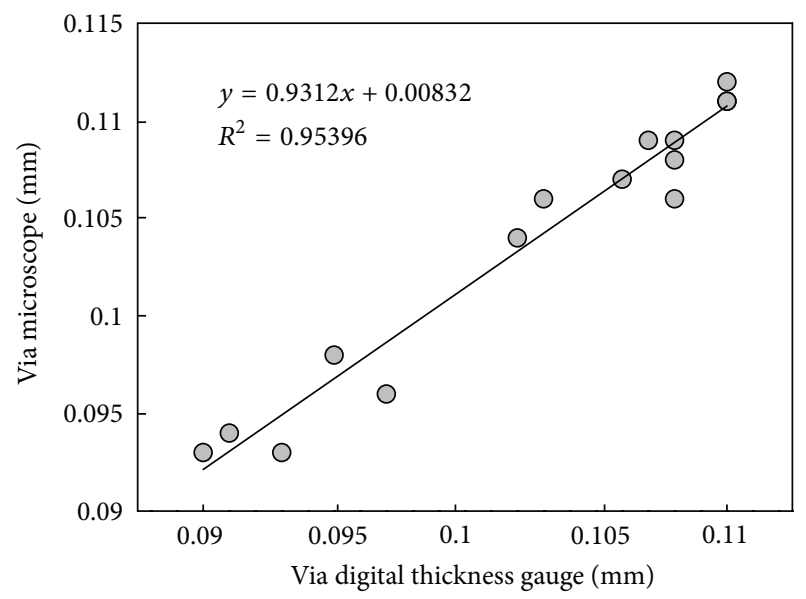

FIGURE 2: The comparison of thickness measured via digital thickness gauge and microscope.

TEMPO, sodium hypochlorite, and sodium bromide system under neutral condition. The oxidation was carried out at $60^{\circ} \mathrm{C}$ for $48 \mathrm{~h}$. Oxidized fibers were thoroughly washed in distilled water and refined in a disk refiner to break apart the residual fiber bundles. The refined nanofibers were then separated by centrifuging at $12000 \mathrm{~g}$ to remove the supernatant fraction and concentrated to $1 \mathrm{wt} \%$ using ultrafiltration. Ultimately, nanofiber suspension was subjected to high mechanical homogenization performed on an $\mathrm{M}$ 110EH-30 Microfluidizer (Microfluidics, Newton, MA, USA) with a series of $200 \mu \mathrm{m}$ and $87 \mu \mathrm{m}$ chambers via a single pass through. The nanofibrils were stocked at $4^{\circ} \mathrm{C}$ without any drying for the subsequent utilization. A typical transmission electron microscopy (TEM) image of TEMPO-oxidized cellulose nanofibril is shown in Figure 1. Such nanofibers have a diameter of approximately $4-10 \mathrm{~nm}$, and a length up to several micrometers.

2.2. Fabrication of $C N F$ and $C N F / P F$ Films. The CNF films were prepared by filtrating CNF suspensions, followed by air-drying and oven-drying. The nanofiber solution, diluted into $0.2 \%$ solids concentration, was filtrated using a $142 \mathrm{~mm}$ Millipore Ultrafiltration System (Millipore, Millipore Corporation, USA) under $0.55 \mathrm{MPa}$ air pressure. Omnipore filter membrane with micropore size of $0.1 \mu \mathrm{m}$ (JVWP14225, JV, Millipore Corporation, USA) was used in the apparatus which was supported by filter paper. The wet films were peeled from the membrane and stacked first between waxed paper and then filter paper, while maintained between two metal plates. The package was air dried at room temperature for 2 days and then oven dried at $60^{\circ} \mathrm{C}$ for $8 \mathrm{~h}$ under a load of $250 \mathrm{~N}$. For preparing CNF/PF composite films, commercially supplied PF resin with $50.5 \%$ solid content (Georgia-Pacific Chemicals LLC, USA) was mechanically mixed with CNF slurries for $1 \mathrm{~h}$, followed by filtration and drying as preparation of CNF films. In this study, the dried composite films with $5 \mathrm{wt} \%$ and $10 \mathrm{wt} \% \mathrm{PF}$ were obtained and named as $\mathrm{CNF} / 5 \% \mathrm{PF}$ and $\mathrm{CNF} / 10 \% \mathrm{PF}$, respectively. Both the oven-dried CNF and CNF/PF films were hot pressed 


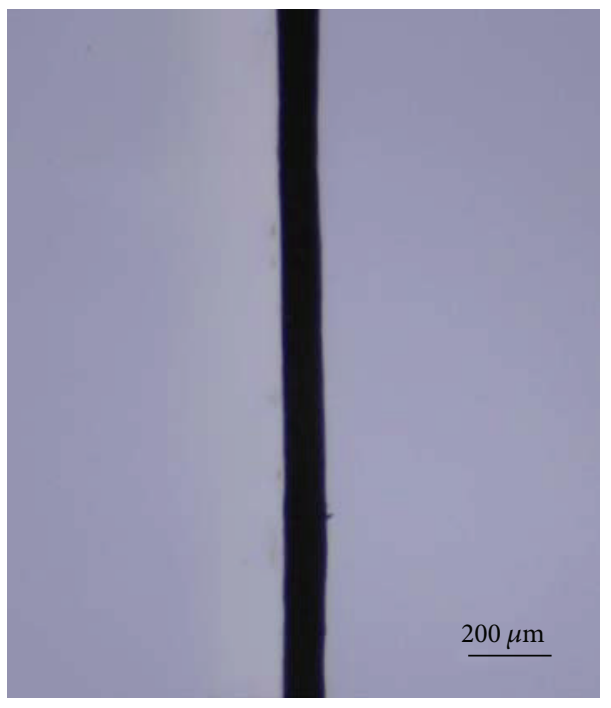

(a)

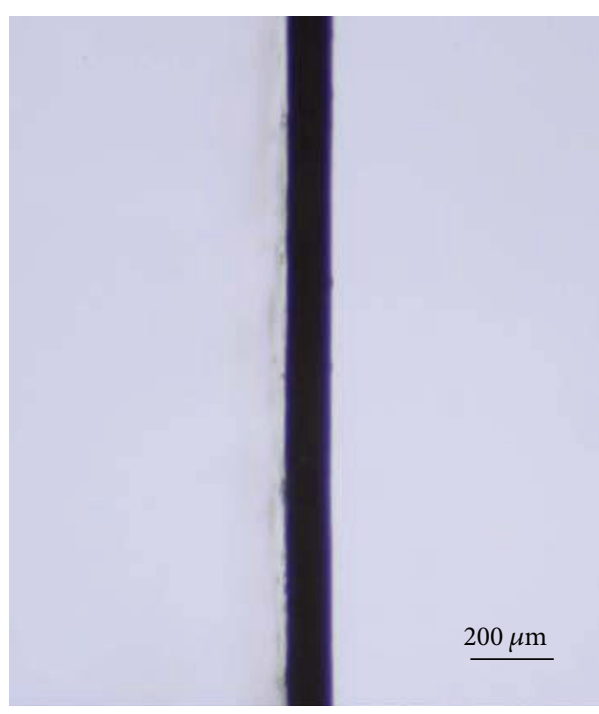

(c)

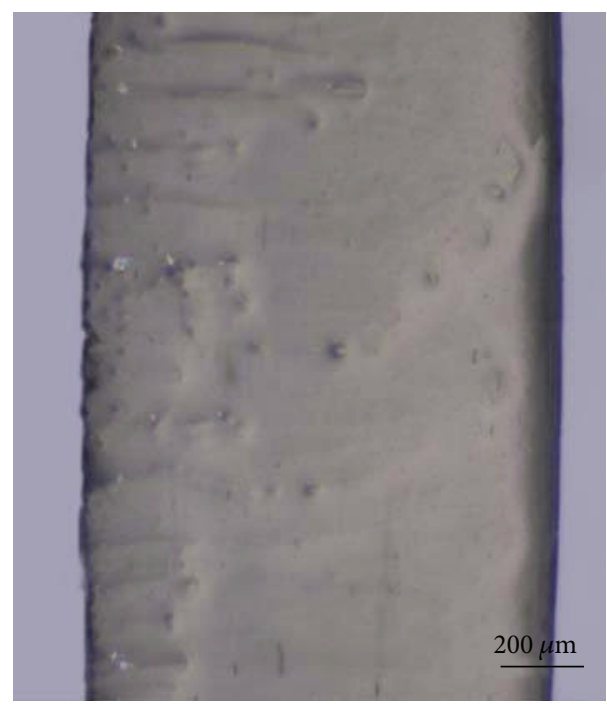

(b)

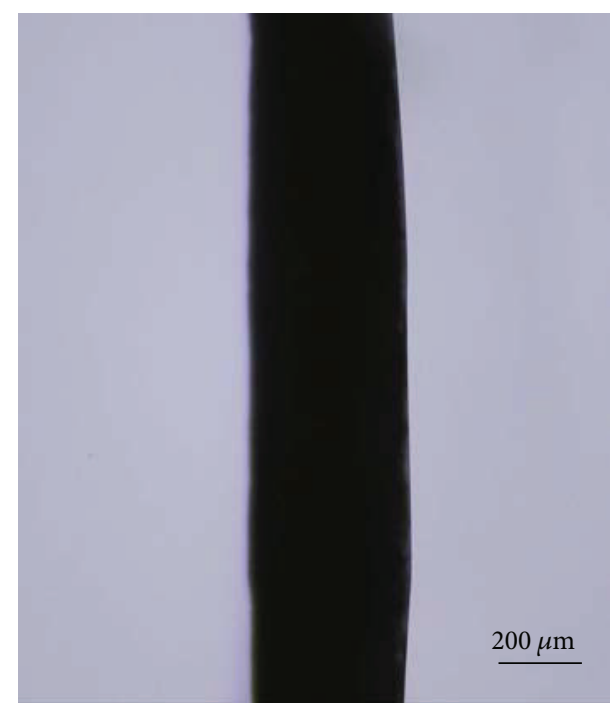

(d)

FIGURE 3: Microscopic cross-section images of CNF (top) and CNF/10\% PF (bottom) films before (left) and after (right) being soaked in water for $5 \mathrm{~min}$. The scale bars represent $200 \mu \mathrm{m}$.

at $130^{\circ} \mathrm{C}$ for $1 \mathrm{~min}$ at $30 \mathrm{MPa}$ of pressure. The films were then conditioned in a $50 \%$ relative humidity chamber at a temperature of $23^{\circ} \mathrm{C}$ until test.

\subsection{Dimensional Expansion Measurement. Rectangular} specimens (30 mm length $\times 1.5-2 \mathrm{~mm}$ width) of CNF and $\mathrm{CNF} / \mathrm{PF}$ films were cut and their initial thicknesses were measured using a digital thickness gauge (Mitutoyo, Mitutoyo Corporation, Japan). The film specimens were then immersed in distilled water and their thickness swelling and width expansion were periodically recorded by taking photos using an optical microscope (SMZ-168T-LED, Motic, USA) equipped with a digital camera (MT3i, Canon, Japan). The magnifications of the ocular and objective lenses were $10 \mathrm{x}$ and $5 \mathrm{x}$, respectively. For the purpose of estimating the actual dimensions of CNF and CNF/PF films, a Bright Line Counting Chamber with exact scales was selected as the reference when pictures were taken. A pair of special holders that was used to assure the observed area was perpendicular to the camera. The exact length of the scale bar illustrated in the referenced image is $200 \mu \mathrm{m}$ and is equivalent to 216 pixels. The microscopic images of all specimens with different dimensions were analyzed with GNU Image Manipulation Program (GIMP, free downloaded from http://www.gimp.org/) software, and thickness or width of each specimen was determined by proportional pixel alignment. Five different locations were measured, and the average value was used to determine the thickness or width. 


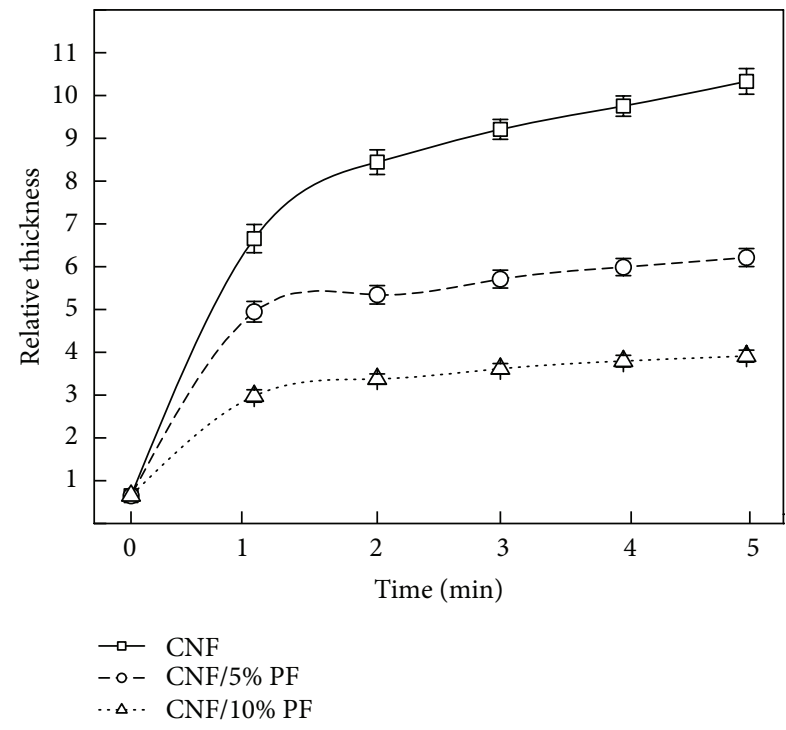

FIGURE 4: Relative thickness variation of CNF and modified CNF films.

\section{Results and Discussion}

3.1. Measurement Value Confirmation. To validate this method, both microscopy image pixel calculation and a standard digital thickness gauge were used to measure several CNF and CNF/PF films of different thickness before being soaked in water. Statistical analysis offers a comparison of the average values produced by such two methods. It can be seen in Figure 2 that the plotted data are highly correlated, approximating a correlation coefficient of 0.95 . The high correlation coefficient indicates that using microscope to measure the dimension of CNF films in water swelling is accurate and reliable. Thus, the method reported here provides a quick and effective measurement of wet/swelled CNF materials.

\subsection{Thickness Swelling of CNF and Modified CNF Films.} Figure 3 illustrates microscopic images on cross-section for $\mathrm{CNF}$ and $\mathrm{CNF} / \mathrm{PF}$ films soaked in water. The relative thickness as a function of time is shown in Figure 4. Here, relative thickness defined as the ratio of current thickness over initial thickness is used to monitor the dimensional variation. Interestingly, both $\mathrm{CNF}$ and $\mathrm{CNF} / \mathrm{PF}$ films exhibited dramatic thickness increase when first exposed to water, despite at different swelling rates. However, their swelling capabilities suddenly decreased in the latter testing interval. After $5 \mathrm{~min}$ immersion, the relative thicknesses for $\mathrm{CNF}, \mathrm{CNF} / 5 \% \mathrm{PF}$, and CNF/10\% PF films swelled approximately to $10.00,6.10$, and 4.00 times, respectively. It should be noted that CNF and $\mathrm{CNF} / \mathrm{PF}$ films have different capacities in absorbing water molecules. Further observation indicates that PF resin has a significantly positive effect on reducing water adsorption in CNF films. These results match well with our previous work in gravimetrically evaluating water absorption of neat and PF modified CNF films [16]. Compared to untreated films, a dramatic reduction of water absorption was observed in $\mathrm{PF}$ modified CNF films.
As expected, the relative thickness for $\mathrm{CNF} / 5 \% \mathrm{PF}$ is 1.5 times greater than $\mathrm{CNF} / 10 \% \mathrm{PF}$ after $5 \mathrm{~min}$ swelling. This convinces that higher resin loadings in composite films will lead to more significant reduction of thickness swelling, as well as water absorption. When examining water absorption of CNF/PF composite films, it was noted that water tended to form small droplets on the surface as PF content approached $30 \%$ [17]. The desirable constraint of water absorption capability resulted majorly from hydrophobic nature of the cured PF resin, and a detailed interpretation is given in the following section. Additionally, each error bar shown in Figure 3 represents 95\% confidence intervals for averaging 5 replicate measurements, indicating the plotted data are in good agreement and of great reproducibility.

\subsection{Width Expansion of CNF and Modified CNF Films.} Microscopic images of the specimen surface are shown in Figure 5. Due to the moistening effect of water, surfaces for both CNF and modified CNF films after water impregnation appear to be smoother than those without any treatment. Relative width calculated from the ratio of current width over initial width is displayed in Figure 6. It can be seen that relative width for $\mathrm{CNF}, \mathrm{CNF} / 5 \% \mathrm{PF}$, and $\mathrm{CNF} / 10 \% \mathrm{PF}$ films after being soaked in water for $5 \mathrm{~min}$ are 1.11, 1.09, and 1.06 times, respectively. Compared to significant thickness swelling, width expansions for both $\mathrm{CNF}$ and $\mathrm{CNF} / \mathrm{PF}$ films are negligible, suggesting the interconnected nanofibrils random-in-the-plane are difficult to swell and separate by the permeation of water molecules.

The swelling difference in thickness and width originates naturally from the deposition of individual nanofibrils during CNF suspension filtration. As illustrated in Figure 1, TEMPO-oxidized nanofibrils in this study are of extremely high aspect ratio. When draining water from the nanofibrils solution, these long nanofibers are most likely to reorganize in a flatwise orientation, owing to the forces of gravity. This phenomenon is evidenced by the microstructure shown in high-resolution scanning electron microscopy (SEM) images. When observed in cross-(or fracture surface) surface, the CNF films were exhibited as a composition of numerous thin layers of nanofilm $[5,17]$. Furthermore, surface plane of CNF films is likely produced by tangling and interlocked individual nanofibrils. The tangling strength and intensity on the plane is much greater than that which is in the spaces. These mechanical interlock effects and structural characteristics cause significant difference of dimensional expansion in plane and space orientation while impregnated in water. Similar findings were also proposed by Svagan et al., who convinced that the intralaminar (on the plane) nanofibrils interactions were stronger than the interlaminars (in space) [18]. In addition, residual growth stress generated in the process of water removal including filtration and oven drying probably contributes to the difference.

3.4. Effect of Modifier on Water Swelling Characteristics. Due to abundant hydrophilic hydroxyls on the nanofibrils, both neat and composite CNF films are sensitive to moisture absorption in high humidity condition $[6,19]$. When exposed 


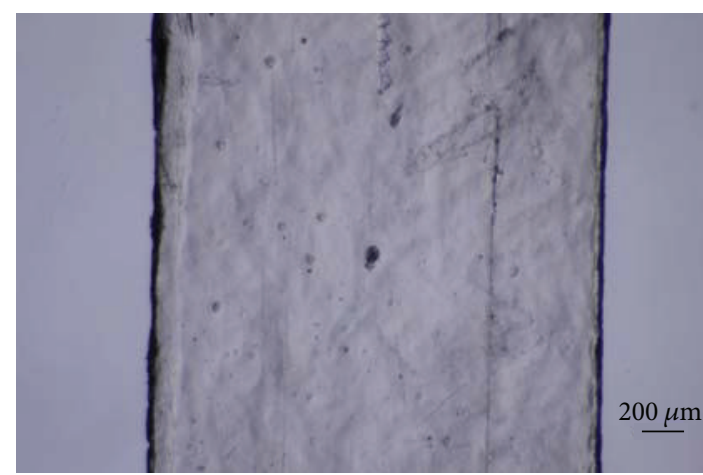

(a)

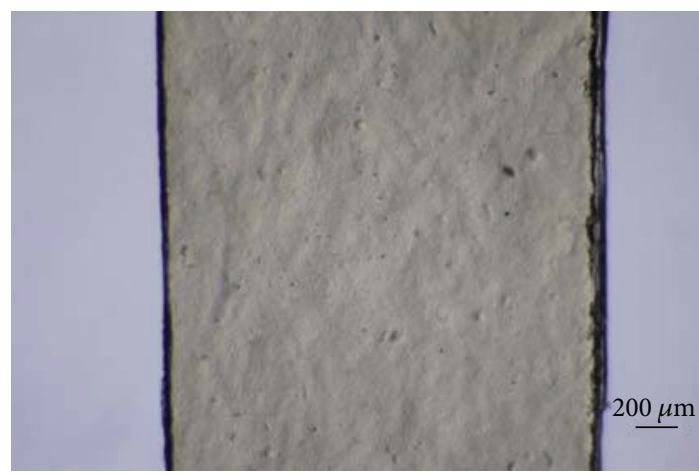

(c)

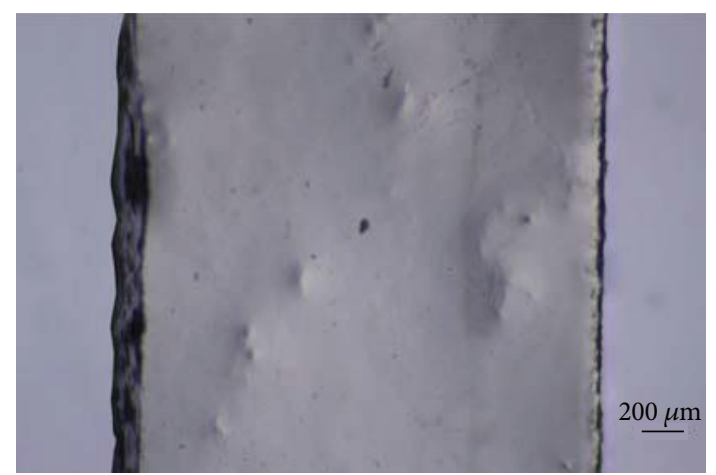

(b)

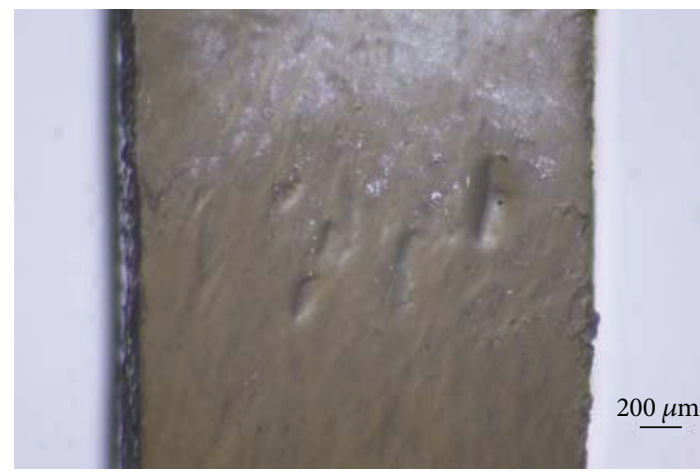

(d)

Figure 5: Microscopic surface images of CNF (top) and CNF/5\% PF (bottom) films before (left) and after (right) being soaked in water for $5 \mathrm{~min}$. The scale bars represent $200 \mu \mathrm{m}$.

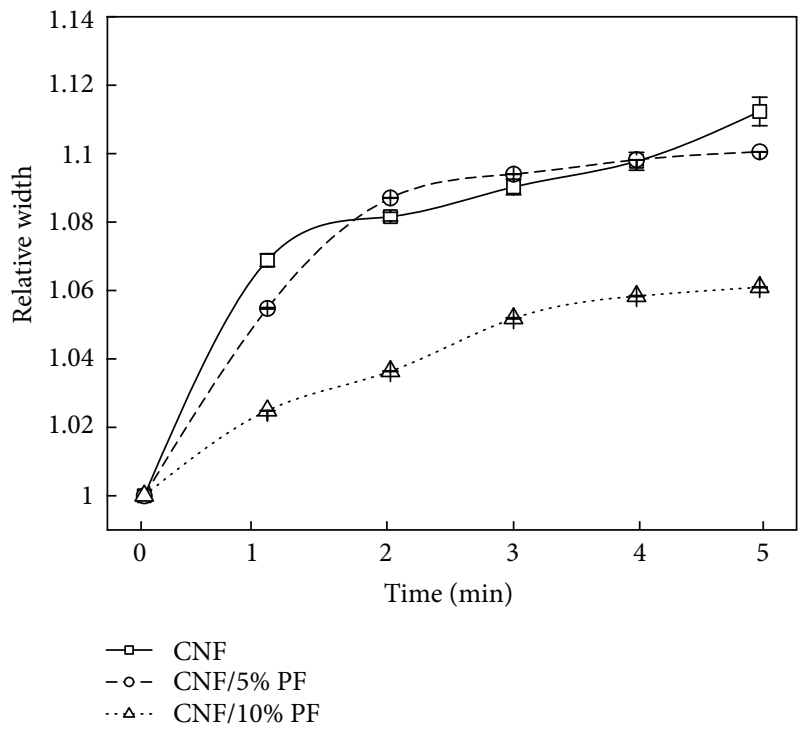

Figure 6: Relative width expansions for CNF and modified CNF films.

to water, the hydrogen $(\mathrm{H})$ bonds between adjacent nanofibrils become partially replaced by CNF-water bonding [19]. As a consequence, the thickness of the film swells dramatically and is accelerated by permeation of water molecule. In the

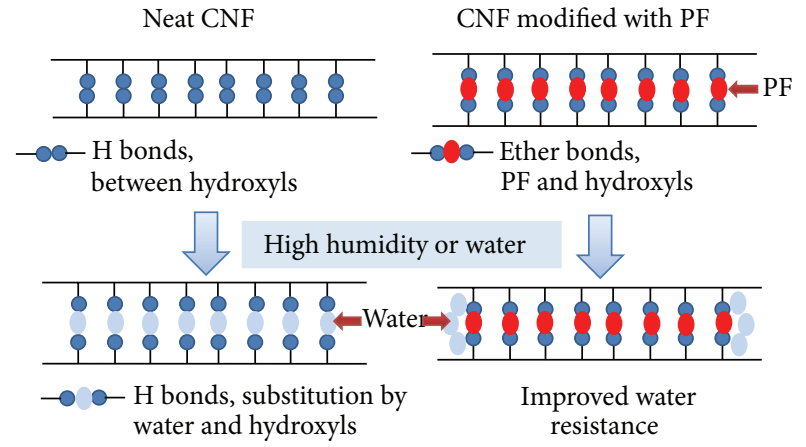

FIGURE 7: Schematic model of $\mathrm{H}$ bonds from adjacent nanofibrils in neat CNF films and ether bonds generated between CNF and modifier PF, and their reaction with water.

PF modified films, however, water resistant bonds like ethers are generated from the reaction between nanofibrils and polymers $[4,20]$. These bonds are resistant to deterioration through exposure to water molecules, resulting in major limitation to water absorption and dimensional swelling. Schematic models of different bonds and their possible reactions with water are summarized in Figure 7.

In addition, the hydrophobic nature and cross-linked structure of cured PF resin plays an important role in cons- 
triction of water swelling [16, 17]. On one hand, PF polymer attaches to the surface of individual nanofibrils. The possibility of hydrophilic hydroxyls being exposed to water molecules is largely reduced. On the other hand, the cross-linked structure of cured PF resin, which serves as a barrier, prevents CNF films from further water swelling. Therefore, the integral effects generated by a water-resistant interface, reduction of feasible hydroxyls, and stability of initial structure contribute to the limitation of thickness swelling for PF modified films.

\section{Conclusions}

Microscope imaging successfully provides a fast and effective way to monitor the instant dimensional changes of CNF and $\mathrm{CNF} / \mathrm{PF}$ films under water immersion. The results showed that the thickness of the films swelled quickly in the first minute and tended to remain stable 5 minutes later. Ultimate relative thickness of CNF, CNF $/ 5 \mathrm{wt} \% \mathrm{PF}$, and $\mathrm{CNF} / 10 \mathrm{wt} \%$ PF films after 5 min immersion increased up to $10.00,6.10$, and 4.00 times, respectively. Nevertheless, width expansions by water for both neat and modified CNF films were negligible. The integral effects of mechanical interlock and residual stress in filtration and drying contribute greatly to the different swelling rates. Addition of water-resistant modifier significantly constrained the thickness swelling, depending on the resin loadings. The water-resistant cured PF resin and the ether bonds formed between hydrophilic hydroxyl groups and $\mathrm{PF}$ resin improved the water resistance of modified CNF films. These films are considered as potential applications in package materials and flexible electronic substrates.

\section{Conflict of Interests}

The authors declare that they have no conflict of interests.

\section{Acknowledgments}

This work was partly financially supported by the National "948" Project of China (2009-4-51). The authors would like to acknowledge Ronald Sabo and Craig Clemons, of the Forest Products Laboratory, for their kind help in microscopy observation and Neil Gribbins for his editing and technical assistance.

\section{References}

[1] F. W. Herrick, R. L. Casebier, J. K. Hamilton et al., "Microfibrillated cellulose: morphology and accessibility," Journal of Applied Polymer Science, vol. 37, no. 9, pp. 797-813, 1983.

[2] A. Turbak, F. W. Snyder, and K. R. Sandberg, "Microfibrillated cellulose, a new cellulose product: properties, uses, and commercial potential," Journal of Applied Polymer Science, vol. 37, no. 9, pp. 815-827, 1983.

[3] G. Nyström, A. Mihranyan, A. Razaq, T. Lindström, L. Nyholm, and M. Strømme, "A nanocellulose polypyrrole composite based on microfibrillated cellulose from wood," Journal of Physical Chemistry B, vol. 114, no. 12, pp. 4178-4182, 2010.

[4] A. N. Nakagaito and H. Yano, "The effect of fiber content on the mechanical and thermal expansion properties of biocomposites based on microfibrillated cellulose," Cellulose, vol. 15, no. 4, pp. 555-559, 2008.

[5] M. Henriksson, L. A. Berglund, P. Isaksson, T. Lindström, and T. Nishino, "Cellulose nanopaper structures of high toughness," Biomacromolecules, vol. 9, no. 6, pp. 1579-1585, 2008.

[6] K. Syverud and P. Stenius, "Strength and barrier properties of MFC films," Cellulose, vol. 16, no. 1, pp. 75-85, 2009.

[7] I. Siró and D. Plackett, "Microfibrillated cellulose and new nanocomposite materials: a review," Cellulose, vol. 17, no. 3, pp. 459-494, 2010.

[8] S. Fält, L. Wågberg, and E. Vesterlind, "Swelling of model films of cellulose having different charge densities and comparison to the swelling behavior of corresponding fibers," Langmuir, vol. 19, no. 19, pp. 7895-7903, 2003.

[9] S. Ahola, J. Salmi, L.-S. Johansson et al., "Model films from native cellulose nanofibrils. Preparation, swelling, and surface interactions," Biomacromolecules, vol. 9, no. 4, pp. 1273-1282, 2008.

[10] C. Aulin, S. Ahok, P. Josefsson et al., "Nanoscale cellulose films with different crystallinities and mesostructures - their surface properties and interaction with water," Langmuir, vol. 25, no. 13, pp. 7675-7685, 2009.

[11] P. Josefsson, G. Henriksson, and L. Wågberg, "The physical action of cellulases revealed by a quartz crystal microbalance study using ultrathin cellulose films and pure cellulases," Biomacromolecules, vol. 9, no. 1, pp. 249-254, 2008.

[12] P. Perré and F. Huber, "Measurement of free shrinkage at the tissue level using an optical microscope with an immersion objective: results obtained for Douglas fir (Pseudotsuga menziesii) and spruce (Picea abies)," Annals of Forest Science, vol. 64, no. 3, pp. 255-265, 2007.

[13] A. Taguchi, K. Murata, and T. Nakano, "Observation of cell shapes in wood cross-sections during water adsorption by confocal laser-scanning microscopy (CLSM)," Holzforschung, vol. 64, no. 5, pp. 627-631, 2010.

[14] R. Masoodi, R. F. EI-Hajjar, K. M. Pillai, and R. Sabo, "Mechanical characterization of cellulose nanofiber and bio-based epoxy composite," Materials and Design, vol. 36, pp. 570-576, 2012.

[15] T. Saito, M. Hirota, N. Tamura et al., "Individualization of nanosized plant cellulose fibrils by direct surface carboxylation using TEMPO catalyst under neutral conditions," Biomacromolecules, vol. 10, no. 7, pp. 1992-1996, 2009.

[16] Y. Qing, R. Sabo, Y. Wu, and Z. Cai, "High-performance cellulose nanofibril composite films," BioResouces, vol. 7, no. 3, pp. 3064-3075, 2012.

[17] Y. Qing, R. Sabo, Z. Cai, and Y. Wu, "Resin impregnation of cellulose nanofibril films facilitated by water swelling," Cellulose, vol. 20, no. 1, pp. 303-313, 2013.

[18] A. J. Svagan, M. A. S. A. Samir, and L. A. Berglund, "Biomimetic polysaccharide nanocomposites of high cellulose content and high toughness," Biomacromolecules, vol. 8, no. 8, pp. 25562563, 2007.

[19] C. Aulin, M. Gällstedt, and T. Lindström, "Oxygen and oil barrier properties of microfibrillated cellulose films and coatings," Cellulose, vol. 17, no. 3, pp. 559-574, 2010.

[20] A. Pizzi and N. J. Eaton, "A conformational analysis approach to phenol-formaldehyde resins adhesion to wood cellulose," Journal of Adhesion Science and Technology, vol. 1, no. 1, pp. 191200, 1987. 

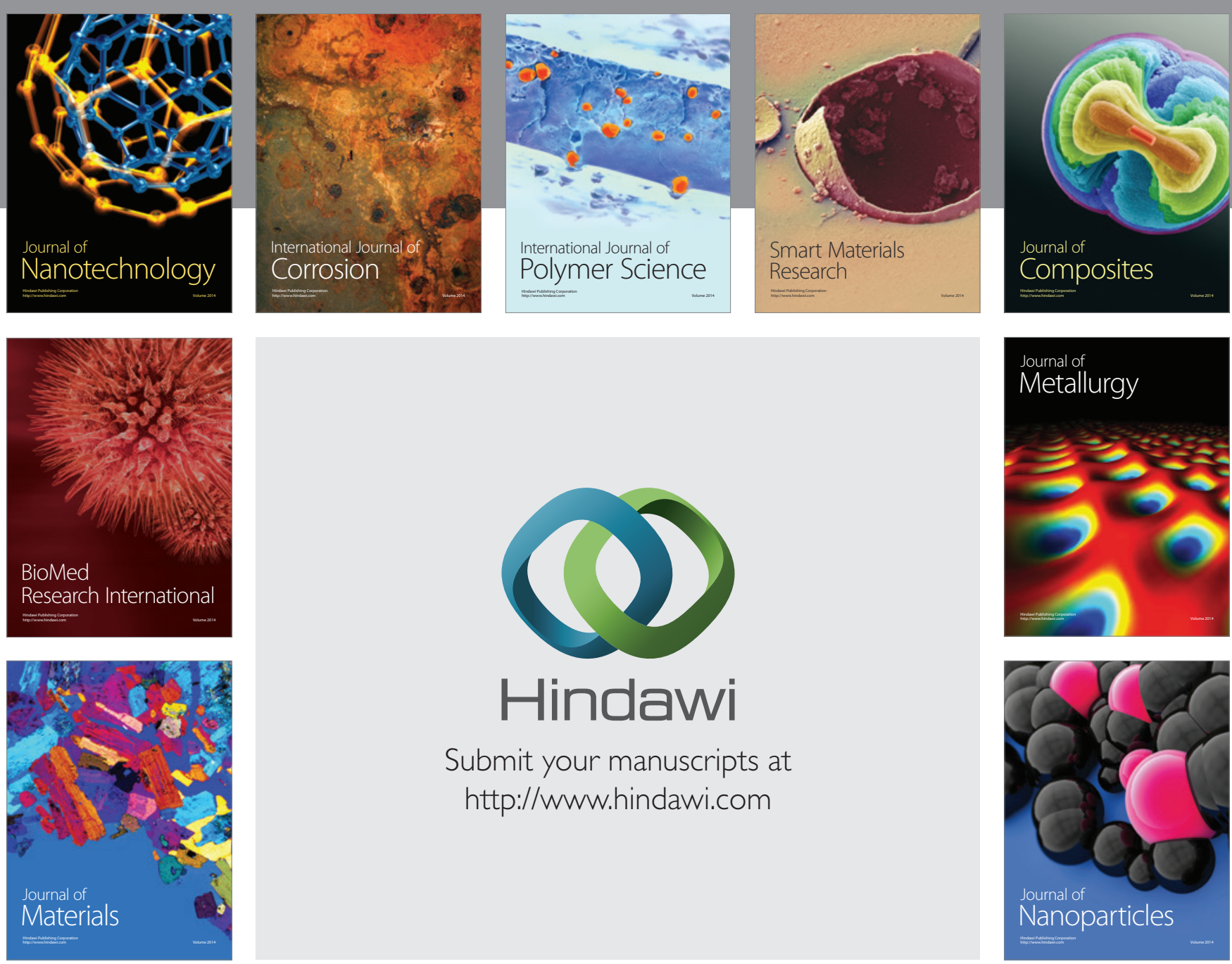

Submit your manuscripts at http://www.hindawi.com
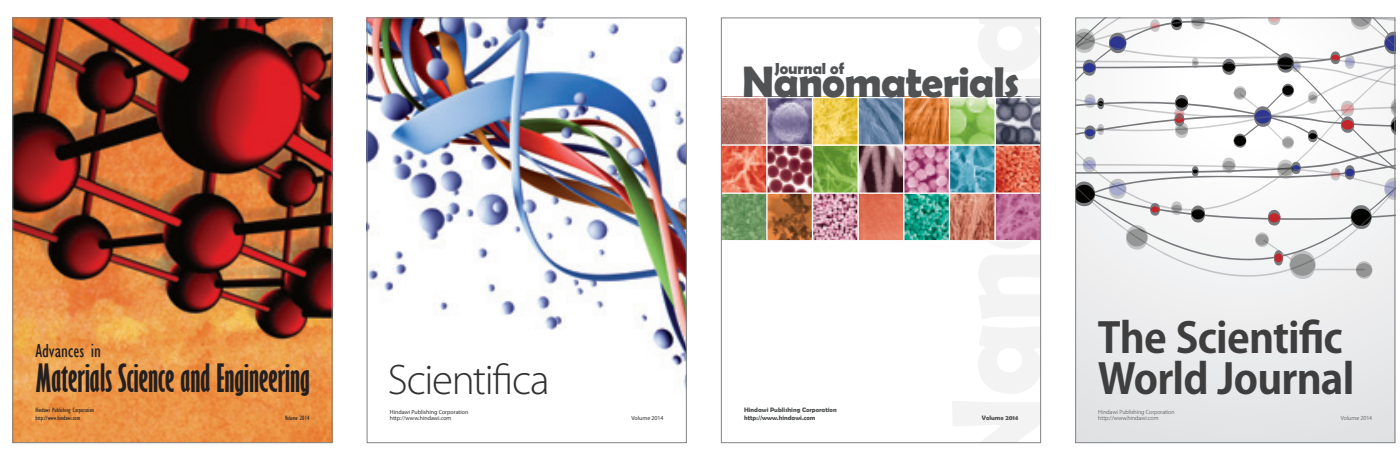

\section{The Scientific World Journal}
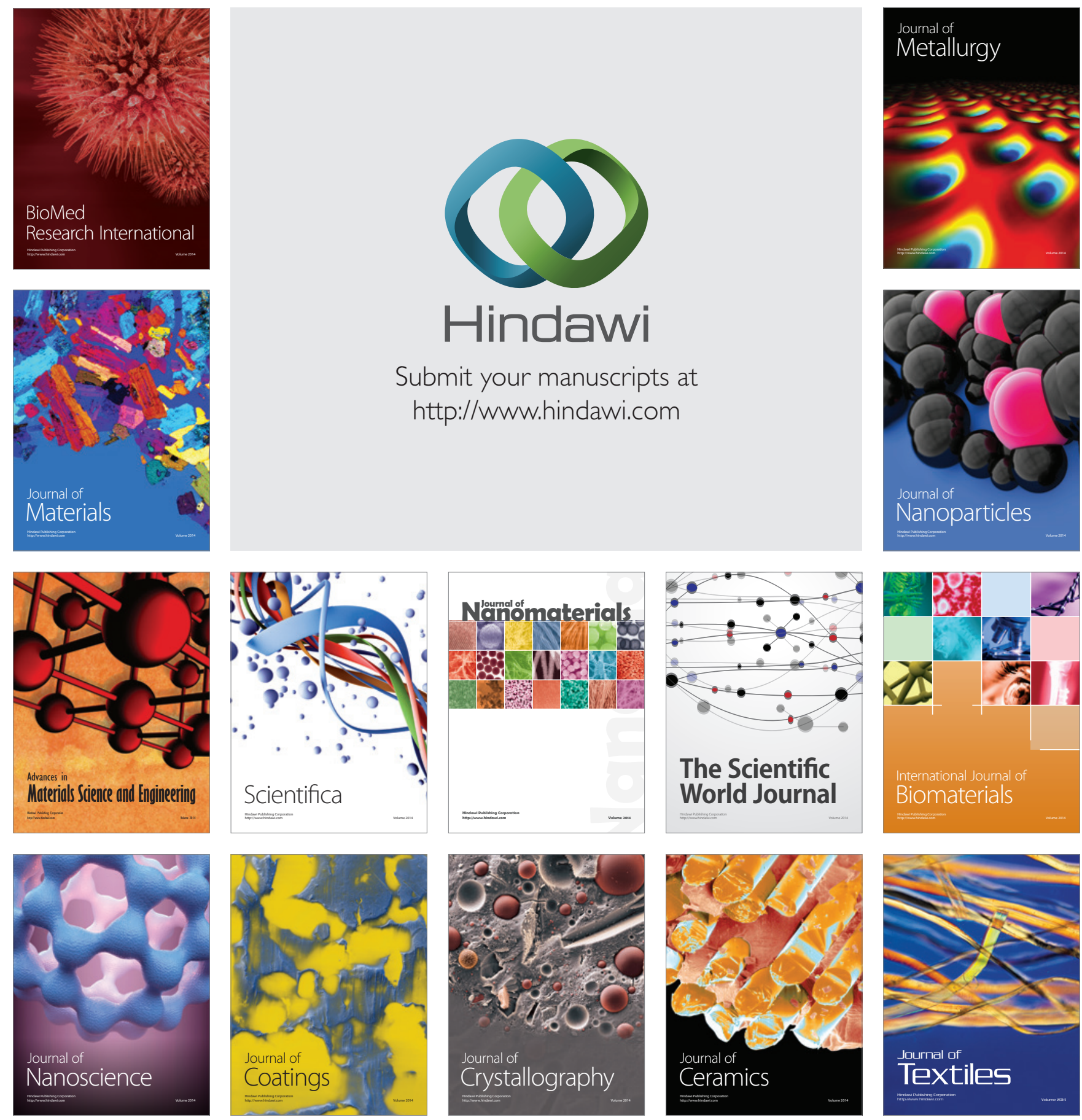